\title{
An in vitro Evaluation of the Hierarchical Micro/Nano Porous Structure of a Ti3Zr2Sn3Mo25Nb Alloy after Surface Dealloying
}

Lan Wang ${ }^{1,2}$, Wenhao Zhou ${ }^{2}$, Zhentao $\mathrm{Yu}^{2}$, Sen $\mathrm{Yu}^{* 2,3}$, Lian Zhou ${ }^{* 1,2}$, Yemin $_{\text {Cao }}^{4}$, Matthew Dargusch ${ }^{5}$, Gui Wang ${ }^{5}$

${ }^{1}$ School of Materials Science and Engineering, Northeastern University, Shenyang 110004, PR China;

${ }^{2}$ Shaanxi Key Laboratory of Biomedical Metal Materials, Northwest Institute for Nonferrous Metal Research, Xi'an 710016, PR China;

${ }^{3}$ East China Jiaotong University, Nanchang, 330013, PR China;

${ }^{4}$ Shanghai Hospital of Integrated Traditional Chinese and Western Medicine,

Shanghai University of Traditional Chinese Medicine, Shanghai, 201203, PR China;

${ }^{5}$ Centre for Advanced Materials Processing and Manufacturing (AMPAM), The

University of Queensland, St Lucia, Queensland 4072 Australia. 


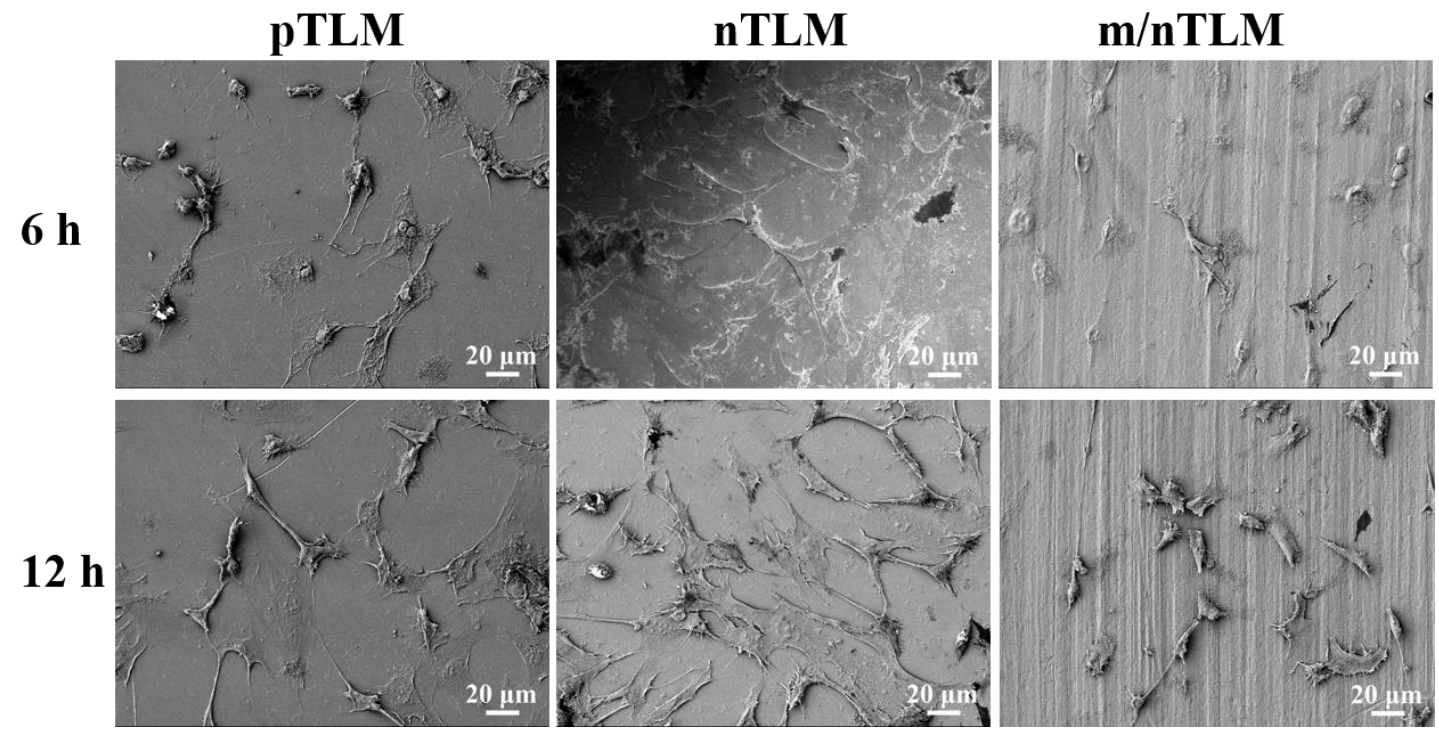

Figure S1. SEM micrograph of the MC3T3-E1 cells cultured on pTLM, nTLM and $\mathrm{m} / \mathrm{nTLM}$ for $6 \mathrm{~h}$ and $12 \mathrm{~h}$.

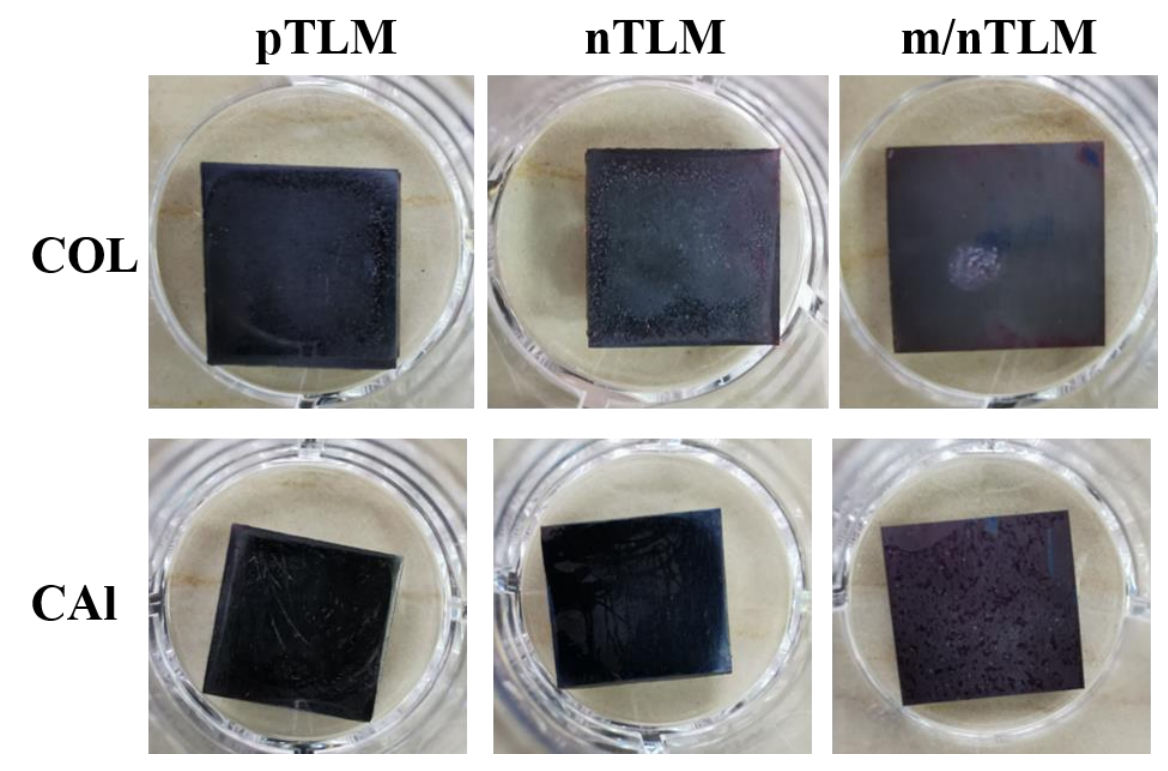

Figure S2. The coloration samples after elution. 